TRABAJOS ORIGINALES

\title{
Selección de hábitat y composición de la dieta de Microlophus occipitalis (Reptilia: Tro- piduridae) en Sechura, Piura - Perú
}

\author{
Habitat selection and diet composition of Microlophus occipitalis (Reptilia: Tropiduridae) in \\ Sechura, Piura - Peru
}

\section{César Chávez-Villavicencio ${ }^{1}$ Yohani Ibáñez-Álvarez ${ }^{2}$, Jesús Manuel Charcape-Ravelo ${ }^{2 *}$}

1 Programa de Doctorado en Biología y Ecología Aplicada. Universidad Católica del Norte. Larrondo 1281, Coquimbo - Chile.

2 Departamento de Ciencias Biológicas. Universidad Nacional de Piura. Campus Universitario, Urb. Miraflores s/n, Castilla- Piura - Perú.

*Autor para correspondencia

Email César Chávez-Villavicencio: cchavez@ucn.cl

Email Yohani Ibáñez-Álvarez:caracucho2004@gmail.com

Email Jesús Manuel Charcape-Ravelo: jcharcaper@unp.edu.pe

\section{Resumen}

\begin{abstract}
Microlophus occipitalis es una lagartija diurna que se distribuye en las costas de Ecuador y Perú, de la cual se pretende determinar el uso y selección de los recursos de hábitat, así como los principales componentes de su dieta. Se trabajó en la laguna Ñapique (Piura, Perú), usando el Diseño Tipo I de Manly (la disponibilidad y uso se estiman para todos los individuos de la especie dentro del área de estudio), y el Coeficiente de Selección de Manly para determinar la selección de recursos. La composición de la dieta y amplitud del nicho trófico se analizó con el índice de Levins estandarizado. Un total de 1055 observaciones permitieron determinar que $M$. occipitalis seleccionó los recursos Tronco y Piedra. La actividad dominante fue la Alimentación, seguida del Soleamiento. Existió un alto consumo de Himenópteros, seguido de los Coleópteros. Microlophus occipitalis es una especie que usa principalmente los troncos y las piedras que se presentan en su hábitat para poder termorregular. Su principal actividad es la alimenticia. Es una especie carnívora que en la temporada de primavera-verano consume principalmente hormigas y coleópteros pero complementa su dieta con flores y hojas en baja cantidad, en un consumo aparentemente accidental.
\end{abstract}

Palabras clave: Ecología Reptiles; Etología; Selección Manly; Termorregulación Heliotérmica; Uso Hábitat.

\section{Abstract}

Microlophus occipitalis is a diurnal lizard distributed on the coasts of Ecuador and Peru, of which it is tried to determine the use and resources selection of habitat, as well as the main components of its diet. Survey was done in Napique lagoon (Piura, Peru), using the Design Type I of Manly (availability and use are estimated for all individuals of the species within the study area), and the Manly Selection Ratio to determine resources selection. The composition of the diet and amplitude of the trophic niche was analyzed with the standardized Levins index. A total of 1055 observations allowed determining that M. occipitalis selected the Trunk and Stone resources. The dominant activity was Food, followed by Sunbathe. There was a high consumption of Hymenoptera, followed by the Coleoptera. Microlophus occipitalis is a species that mainly uses the trunks and stones that occur in its habitat to be able to thermoregulate. Its main activity is food. It is a carnivorous species that in the spring-summer season consumes mainly ants and coleoptera but complements its diet with flowers and leaves in low quantity, in a seemingly accidental consumption.

Keywords: Habitat Use; Manly Selection Ratio; Reptiles Ecology; Ethology; Heliothermic Thermoregulation.

Citación:

Chávez-Villavicencio C., Y. Ibáñez-Álvarez, J.M. Charcape-Ravelo. 2018. Habitat selection and diet composition of Microlophus occipitalis (Reptilia: Tropiduridae) in Sechura, Piura - Peru. Revista peruana de biología 25(3): 221 - 228 (Agosto 2018). doi: http://dx.doi.org/10.15381/ rpb.v25i3.13403

$\begin{array}{ll}\text { Presentado: } & 08 / 07 / 2017 \\ \text { Aceptado: } & 10 / 05 / 2018 \\ \text { Publicado online: } & 25 / 09 / 2018\end{array}$

Información sobre los autores:

CC-V, YI-A, MC-R: Diseñaron el estudio, probaron el diseño, analizaron datos, redactaron, revisaron y aprobaron manuscrito YI-A: Tomó datos en campo.

Los autores no incurren en conflictos de intereses. 


\section{Introducción}

Microlophus occipitalis es una lagartija de hábitos diurnos, trepadora, y forrajea en el suelo (Dixon \& Wright 1975, Venegas 2005, Jordán \& Pérez 2012). Se distribuye en las costas occidentales de Ecuador y Perú, desde el nivel del mar hasta los $700 \mathrm{msnm}$, y es registrada cerca a pequeños peñascos, árboles, arbustos, en playas con matorrales densos, bosques semiáridos, incluso bajo escombros de viviendas (Dixon \& Wright 1975, Watkins 1977). Esta especie controla su temperatura cambiando de posición de acuerdo a la hora del día, por la mañana y tarde usan rocas o espacios abiertos para calentarse, y al medio día prefieren lugares con sombra como árboles o arbustos (Jordán \& Pérez, 2012).

A pesar de ser una especie muy frecuente, no se registra información sobre selección de hábitat y la composición de su dieta. Es de particular interés poder determinar qué recursos del hábitat son seleccionados más frecuentemente que otros, porque provee información fundamental sobre la naturaleza de los animales y el modo en que ellos satisfacen sus necesidades de supervivencia (Manly et al. 2003). Los estudios sobre la selección de recursos proveen información cuantitativa, que a su vez, es un indicador de los requerimientos a largo plazo de una población, y son útiles para proyectar el impacto del cambio en el hábitat y poder definir la capacidad de carga hipotética, basada en valores de hábitat bajo ciertas condiciones (Manly et al. 2002).

Por otro lado, conocer la dieta de una especie animal, permite entender las condiciones del entorno de los organismos, debido a que la estructura trófica está sujeta a las fluctuaciones ambientales, por lo que las especies están obligadas a aprovechar al máximo el alimento en tiempos y espacios de mayor productividad (Altamirano et al. 2001). No obstante, se conoce que dentro del género Microlophus, $M$. peruvianus, $M$. trigis y $M$. theresiae, se alimentan principalmente de insectos, otros artrópodos, partes vegetales, invertebrados y algas de origen marino propios de la zona intermareal, lo que refleja que estos saurios costeros presentan una dieta omnívora, mostrando una tendencia a una elevada amplitud de nicho trófico así como su capacidad para actuar como depredadores oportunistas (Dixon \& Wright 1975, Vargas 2000, Catenazzi 2002, Pérez \& Balta 2007, Pérez et al. 2008, Quispitúpac \& Pérez 2009).

Debido a que los estudios sobre $M$. occipitalis han sido enfocados en su taxonomía, distribución, abundancia (Carrillo \& Icochea 1995, Dixon \& Wright 1975, Venegas 2005, Viera 2007) y comportamiento reproductivo (Watkins 1998), con esta investigación se aporta al conocimiento sobre su ecología en relación al hábitat planteándose como objetivos determinar el uso y selección de los recursos de hábitat de M. occipitalis, así como los principales componentes de su dieta.

\section{Material y métodos}

Área de estudio.- El trabajo se realizó en el sector oeste de

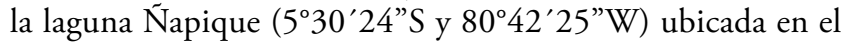
poblado de Cerritos, distrito de Cristo Nosvalga, provincia de Sechura, departamento de Piura. Según el mapa ecológico del Perú, esta localidad pertenece a la zona de vida de desierto superárido Premontano Tropical (ds-PT) (CDC-UNALM 1992). De acuerdo con la clasificación de las comunidades de vegetación del norte del Perú (Mostacero et al. 1996) el área de estudio se encuentra entre dos comunidades: las comunidades
Macrotérmicas o Xerofíticas representada por el algarrobal, propio de la costa septentrional del Perú, donde predomina Prosopis pallida "algarrobo"; y las comunidades de Suelo Salino, representada por los gramadales, también conocidos como "Formación Halófita" (Weberbauer 1945, Ferreyra \& Tovar 1955) y "Estepas Graminosas" (Angulo 1955), donde predomina Distichlis spicata "grama salada”. El algarrobal está conformado principalmente por dos estratos, uno arbóreo y otro herbáceo (Mostacero et al. 1996). El estrato arbóreo está representado por $P$. pallida y el herbáceo está representado frecuentemente por Poáceas, Fabáceas, Amarantáceas, entre otras familias (Mostacero et al. 1996).

Registro de datos.- Se empleó la extensión Animal Movement del programa Arc View Gis 3.3 (ESRI 2002), para establecer en el área de estudio de manera aleatoria 30 parcelas de $20 \mathrm{~m}$ x $20 \mathrm{~m}$, en el algarrobal, cubriendo una extensión de $12 \mathrm{mil} \mathrm{m}^{2}$ de área evaluada. Los datos de uso y disponibilidad de los recursos que conformaron el hábitat de $M$. occipitalis, se colectaron siguiendo el Diseńo Tipo I de Manly donde la disponibilidad y uso se estiman para todos los individuos de la especie dentro del área de estudio (Manly et al. 2003). Este diseño asume independencia en las observaciones y la disponibilidad y uso de cada recurso es igual para todos los individuos, entendiéndose por recursos a los elementos que comparten características similares en una misma área (Manly et al. 2003).

Para este estudio, se consideró como recursos del hábitat a:

- Ramas (R: Ramas grandes de algarrobo que se obtienen por la tala, la construcción de cercos y las que formaron montículos dispersos sobre el área de estudio).

- Herbáceas (H: ya sean vivas o secas pero que se mantuvieron plantadas, pueden ser altas o tipo césped).

- Arena (A: sustrato arenoso).

- Vegetación Arbórea y Arbustiva (VAA: se refiere al algarrobo en su forma de vida arbórea o arbustiva).

- Tronco (T: se refiere al tronco de algarrobo después de ser talado y que se encuentra expuesto al sol).

- Piedra (P: suelo pedregoso y piedras dispersas sobre los otros elementos del suelo).

- Heces y Hojarasca (H-H: se refiere principalmente a restos de hojas del algarrobo y de herbáceas que se encuentran formando pequeños y grandes montículos en el suelo, las heces de animales silvestres y pequeñas ramas secas provenientes de herbáceas o de algarrobo).

El número de usos del recurso fue el número de veces que un individuo se localizó sobre este, mientras que la disponibilidad se determinó por el porcentaje de la superficie de cada tipo de recurso respecto de la superficie total de las parcelas establecidas en el diseño (Manly et al. 2003).

Los datos de uso se registraron entre las 9:00 y 15:00 horas según recomiendan Pérez \& Balta (2007), una vez por mes, desde agosto de 2009 hasta marzo de 2010. Cada parcela fue recorrida por la parte central, desde donde se fue registrando los individuos de $M$. occipitalis en cada recurso y la actividad que realizaron. Las actividades consideradas fueron alimentación: cuando se le observó ingiriendo una presa, soleamiento: 
cuando se le observó tomando sol o termoregulando, descanso: cuando se le observó en reposo bajo sombra, alerta: cuando se le observó en situación de vigilancia o atención, escape: cuando corrió raudo del punto donde fue observado, agresividad: cuando sostuvo enfrentamiento con otro individuo de su especie y otros (caminar: desplazamiento horizontal sobre sustrato, cavar: removiendo tierra para construcción de madriguera y trepar: desplazamiento vertical principalmente en troncos arbustivos y arbóreos). Cada observación tuvo una duración de dos minutos por individuo dentro de cada parcela (Watkins 1998, Vargas 2000, Wareham 2005), es decir, si dentro de la parcela hubo dos individuos el tiempo de observación fue de cuatro minutos.

Para determinar la composición de la dieta, se capturaron temporalmente 36 individuos de $M$. occipitalis (seis hembras y 30 machos), con la ayuda de un lazo (Eakin 1957). No se preservó ningún individuo. Las capturas se realizaron entre agosto y diciembre de 2009, desde las 11:00 hasta las 16:00 horas. El contenido estomacal se obtuvo mediante un lavado de estómago o regurgitación forzada dado que este método permite obtener el contenido estomacal de los saurios sin tener que sacrificarlos. El lavado estomacal se efectuó con un catéter plástico conectado a una jeringa con $60 \mathrm{~cm}^{3}$ de agua (Legler \& Sullivan 1979, Martin \& Hockey 1993, Rivas et al. 1996, Catenazzi 2002). Una vez obtenida la muestra, los individuos evaluados fueron liberados inmediatamente en el lugar donde se observó antes de su captura, para minimizar perturbación. Los individuos no presentaron afectación significativa por los lavados estomacales realizados, a juzgar por su estado general y actividad. Las muestras estomacales fueron conservadas en alcohol al 70\% y analizadas en el laboratorio con la ayuda de un estereoscopio Labomed ${ }^{\oplus} 40 \mathrm{X}$. El análisis de la composición de la dieta se basó en individuos relativamente completos y partes como cabezas que pudieron determinarse y contarse.

Análisis de datos.- La selección de recurso se calculó con coeficiente de selección de Manly (Wi) que indica selección a favor cuando su valor es mayor a 1 , no selección cuando su valor es 1 y selección en contra si su valor es menor a 1 (Manly et al. 2003). La función evalúa la selección de hábitat con el Khi2 de Pearson y el log-likelihood Khi2: Prueba de uso aleatorio de recursos según el estadístico de Pearson. Para los Diseños Tipo I, los resultados del Chi-Cuadrado prueban la hipótesis de que las proporciones de selección son en promedio iguales a cero. (Manly et al. 2003). La precisión de los estimados se verificó con el intervalo de confianza de Bonferroni al 95\% por ser más riguroso que el valor de p de acuerdo a lo expuesto por Johnson (1999) y Sarria \& Silva (2004). El cálculo se realizó usando el paquete adehabitat del programa $\mathrm{R}$ versión 2.11.1 ( $\mathrm{R}$ Development Core Team, 2009). Las actividades realizadas por los individuos se ordenaron de mayor a menor y se graficó su proporcionalidad, de acuerdo al recurso usado.

La composición de la dieta se analizó siguiendo a Pérez \& Balta (2007) que establecen la importancia de cada presa en porcentaje utilizando las siguientes formulas:

$\mathrm{N}=$ (Número de individuos de un tipo de presa/Total de individuos de todas las presas) ${ }^{*} 100$

$\mathrm{F}=$ (Número de estómagos en los que aparece un tipo de presa/Número totales de estómagos) ${ }^{*} 100$

La amplitud del nicho trófico de se analizó con el índice de Levins (1968) estandarizado por Hulbert (1978) mediante las fórmulas:

$$
\begin{gathered}
B=1 / \sum\left(p_{i}\right)^{2} \\
B_{e s t}=(B-1) /(N-1)
\end{gathered}
$$

Donde $B=$ índice de Levins, $p_{i}=$ proporción de presas en total de la muestra, $N=$ Número total de presas y $B_{e s t}=$ índice de Levins estandarizado (escala de $0=$ mínima amplitud de nicho a 1 = máxima amplitud de nicho) (Krebs 1989).

\section{Resultados}

Se realizaron 1055 registros de uso del hábitat que condujeron a determinar que $M$. occipitalis seleccionó los tipos de cobertura Tronco y Piedra sobre Herbáceas, Heces-Hojarasca y Ramas, mientras que los elementos Arena y Vegetación Arbórea-Arbustiva fueron seleccionadas en contra a pesar de su uso (Tabla 1. Fig. 1 ).

Sobre las actividades realizadas por los individuos, las principales fueron las de alimentación (28\%) y soleamiento (21\%) (Fig. 2).

La alimentación se realizó principalmente sobre el recurso Herbáceas y en menor cantidad sobre los otros recursos, pero nunca se observó sobre el recurso Piedra. El soleamiento se realizó principalmente sobre los recursos Tronco y Piedra, y en esta última es la única actividad observada. Las actividades de agresión se realizaron en los recursos Ramas, Arena y Herbáceas y las de alerta incluyeron además Vegetación Arbórea-Arbustiva

Tabla 1: Disponibilidad y Uso por tipo de recurso en Microlophus occipitalis. Coeficientes de Selección de Manly correspondientes a los datos agrupados (Wi= Coeficiente de Selección de Manly. EE= Error Estándar).

\begin{tabular}{lccc}
\hline Tipo de cobertura & Disponibilidad (\%) & Uso & Wi (EE) \\
\hline Ramas & 23.6 & 295 & $1.2(0.5)$ \\
Herbáceas & 13.5 & 200 & $1.4(0.1)$ \\
Vegetación Arbórea-Arbustiva & 26.1 & 99 & $0.4(0.1)$ \\
Arena & 24 & 228 & $0.9(0.1)$ \\
Tronco & 0.5 & 45 & $8.5(0.2)$ \\
Piedra & 0.3 & 18 & $5.7(0.2)$ \\
Heces-Hojarasca & 12 & 170 & $1.3(0.1)$ \\
\hline Total & 100 & 1055 & \\
\hline
\end{tabular}




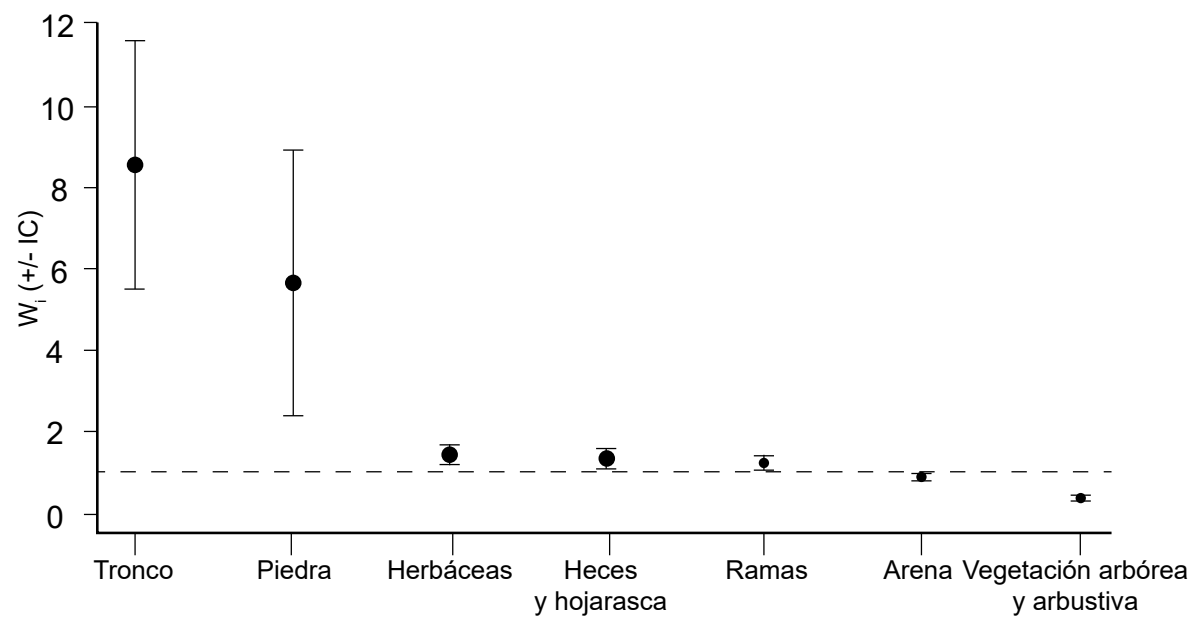

Figura 1. Coeficiente de Selección de Manly (Wi) y sus intervalos de confianza al $95 \%$ por tipo de recurso empleado por Microlophus occipitalis. Piura - Perú. 2010 .

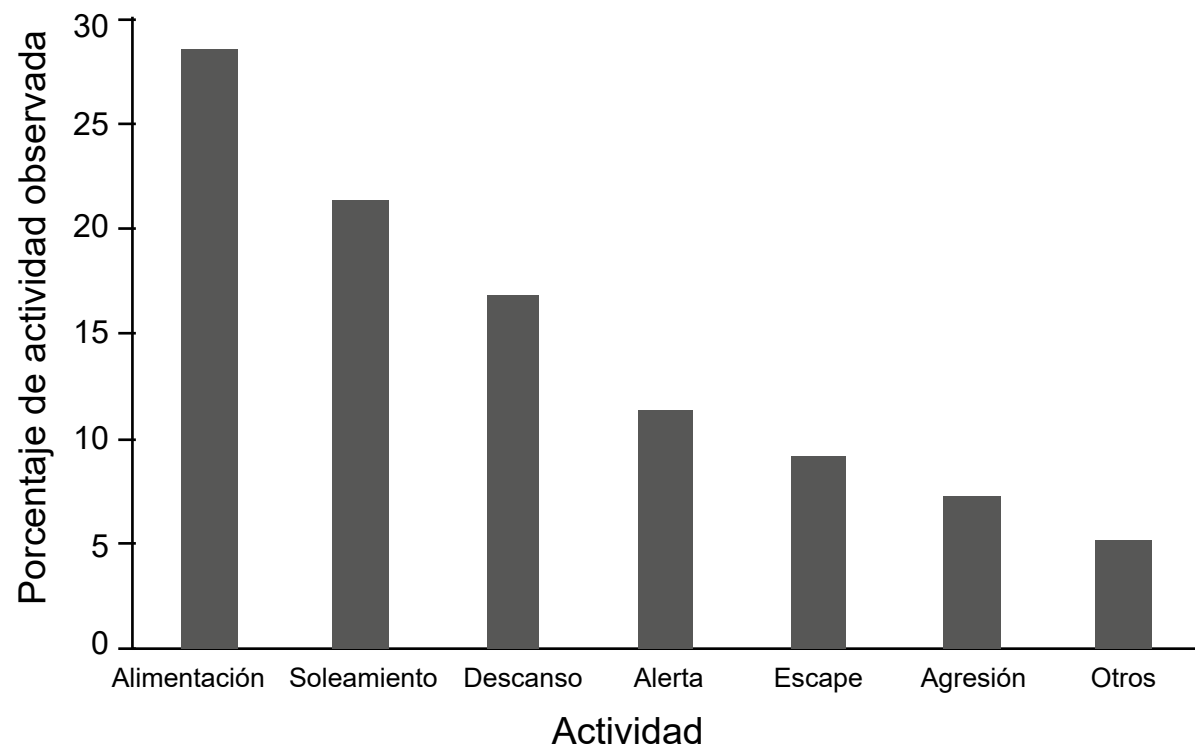

Figura 2. Proporción de las actividades realizadas por Micorlophus occipitalis a partir de 1055 registros de uso.

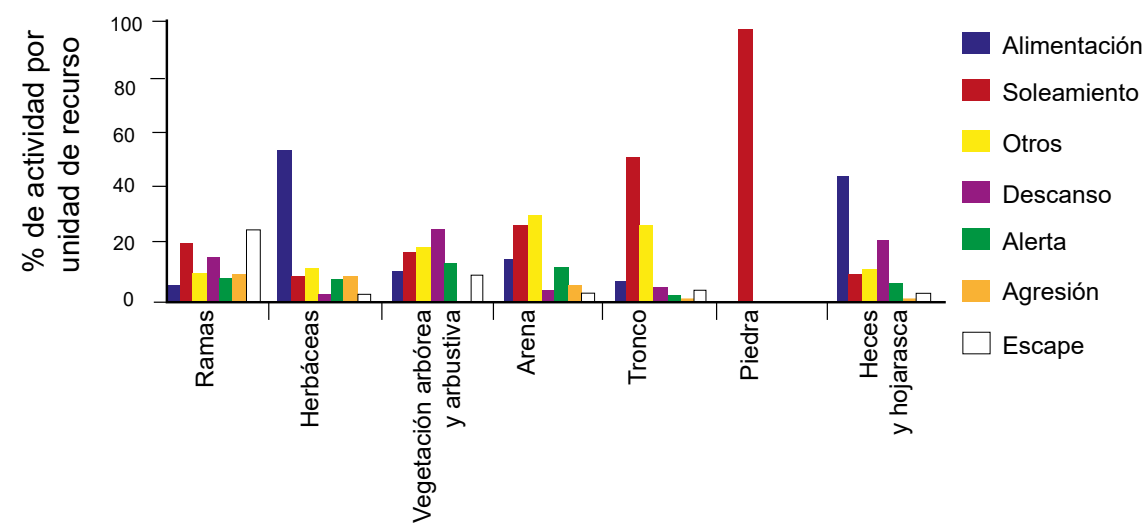

Recursos

Figura 3. Porcentaje de las actividades realizadas por Microlophus occipitalis en cada unidad de recurso. 
y Heces-Hojarasca. El descanso se realizó principalmente en Vegetación Arbórea-Arbustiva, en Heces-Hojarasca y Ramas. Las actividades clasificadas como otros son la tercera actividad más realizada y se presentó en todos los recursos considerados en este estudio, que conformaron el hábitat de la especie (Fig. 3).

Respecto a la dieta, se encontraron 606 presas que se agruparon en 14 ítems correspondientes a nueve Órdenes de insectos, tres Órdenes de arácnidos, larvas de lepidópteros y dípteros, y restos de hojas y flores (Tabla 2).

Existió un alto consumo de Himenópteros $(\mathrm{N}=58.9 \%, \mathrm{~F}$ $=91.7 \%$ ) conformados exclusivamente por hormigas, seguido de los Coleópteros ( $\mathrm{N}=15.2 \%, \mathrm{~F}=80.6 \%)$. El ítem menos consumido fue Odonata (solo se encontró restos correspondientes a un ejemplar). Las flores fueron la estructura vegetal más consumida $(\mathrm{N}=40)$ incluso superando a otros invertebrados. Otros componentes encontrados en el estómago fueron arena y restos de carbón. Estos hallazgos permitieron establecer que la amplitud de nicho trófico fue baja $\left(B_{e s t}=0.13\right)$.

\section{Discusión}

La selección de los recursos Tronco y Piedra se relacionó con el Soleamiento de $M$. occipitalis, que fue la mayor actividad que realizó la especie durante este estudio, dado que estos recursos se encontraron expuestos directamente al sol (nunca estuvieron bajo sombra). Esto coincide con la selección realizada por otras especies de reptiles, principalmente porque sobre las piedras se produce la mejor acumulación del calor y presenta alta conductividad térmica (Clauser \& Huenges 1995), permitiendo mantener las temperaturas corporales óptimas para tener un buen rendimiento en sus procesos fisiológicos, búsqueda de alimento, reproducción y de escape de los depredadores (Huey 1982). Por lo tanto, la selección de estos dos tipos de recursos estuvo dirigida principalmente por la función termorreguladora de la especie (Heath 1964, Huey \& Pianka 1977, Huey 1982). Estos recursos, importantes para la termorregulación, fueron usados en forma desproporcional a su disponibilidad y por lo tanto seleccionados por encima de los otros recursos.

El uso de los recursos Arena y Herbáceas estaría dado porque también ofrecen un medio térmico favorable para las lagartijas por el aire caliente que envuelve la superficie de estos elementos ("boundary layer effect") y, la radiación térmica de estas permite incrementar también sus tasas de calentamiento (Bakken 1989), pero no en la misma magnitud que los recursos Troncos y Piedras. Las diferencias entre estos recursos se basarían en la mayor conductividad de la Arena sobre las Herbáceas por lo que el uso de uno u otro tipo de recurso dependería de la necesidad de ganar y perder calor en elementos más fríos o calientes, propiciados por las variaciones ambientales durante el día. Sin embargo el usar estos recursos tendría una desventaja en la protección contra los depredadores por exponerlas a la detección de estos (Martin 1992).

Los principales recursos usados para obtener el alimento fueron Herbáceas y Heces-Hojarasca, en estos recursos habitan insectos y artrópodos, que son el principal componente de la dieta de la especie (Brennan 2010). A pesar que la alimentación es importante para cualquier organismo, para $M$. occipitalis seleccionar los recursos Tronco y Piedras (relacionadas con la termorregulación) resultó ser más significativo, tal vez debido a
Tabla 2. Dieta de Microlophus occipitalis en un sector del desierto de Sechura (Piura-Perú). Para cada ítem alimentario se indica el número de presas $(\mathrm{N})$, frecuencia $(\mathrm{F})$ y sus respectivos porcentajes $(\mathrm{n}$ presas $=606$. $\mathrm{n}$ estómagos $=36$ ).

\begin{tabular}{lcccc}
\hline Ítem alimenticio & N & \% & F & $\%$ \\
\hline Himenóptera & 357 & 58.9 & 33 & 91.7 \\
Coleóptera & 91 & 15.0 & 29 & 80.6 \\
Homóptera & 26 & 4.3 & 17 & 47.2 \\
Díptera & 22 & 3.6 & 14 & 38.9 \\
Lepidóptera & 6 & 1.0 & 6 & 16.7 \\
Hemíptera & 11 & 1.8 & 8 & 22.2 \\
Dermáptera & 10 & 1.7 & 8 & 22.2 \\
Ortóptera & 5 & 0.8 & 5 & 13.9 \\
Odonata & 1 & 0.2 & 1 & 2.78 \\
Aranae & 16 & 2.6 & 14 & 38.9 \\
Scorpionida & 3 & 0.5 & 3 & 8.33 \\
Pseudoscorpionida & 3 & 0.5 & 2 & 5.56 \\
Larvas de Insectos en general & 5 & 0.8 & 4 & 11.1 \\
Flores y hojas & 43 & 7.1 & 4 & 11.1 \\
\hline
\end{tabular}

que si estos animales no tienen la temperatura corporal adecuada difícilmente conseguirían su alimento.

La selección de recursos de la especie parece estar dirigida básicamente por la termorregulación, la cual permite cumplir funciones importantes como la alimentación. Bartholomew (1982) estableció que los reptiles realizan la termorregulación por mecanismos conductuales seguido de mecanismos fisiológicos. Cualquiera fuera el mecanismo que emplea M. occipitalis, la termorregulación sigue siendo el proceso más importante para la especie. Pearson (1977) demostró la relevancia de la selección del sustrato en la termorregulación de Liolaemus signifier, una especie de lagarto que habita a $4200 \mathrm{~m}$ de altitud, que se calienta más rápido al usar perchas de baja inercia térmica (ramas de arbustos), antes que perchas con alta inercia (rocas), como lo hacen la mayoría de las especies de Liolaemus de Chile central (Carothers et al. 1998).

Otro aporte significativo de este trabajo es que permite alertar sobre los cambios conductuales que se ejercen sobre la fauna. Microlophus occipitalis existe hace 5 millones de años en la tierra (Heise 1998), cuando en su hábitat no se encontraban considerables cantidades de árboles talados. ¿Una actividad antrópica como la tala estaría determinando que estos lagartos realicen sus actividades de termorregulación sobre restos de troncos, cambiando de alguna manera su conducta ancestral? De ser cierta esta afirmación, este cambio en el comportamiento estaría demostrando la elasticidad de la especie para adaptarse a vivir en ambientes altamente perturbados, lo cual resultaría favorable para su supervivencia.

En cuanto a la dieta de $M$. occipitalis, el ítem Himenóptera presentó una notable diferencia con respecto de los otros ítems. Es posible que esta especie ingiera gran cantidad de hormigas (Hymenoptera) debido a que son presas muy pequeńas por lo que necesitaría mayor cantidad para compensar el poco volumen 
que presentan, para llenar sus estómagos. Considérese que los otros ítems tienen un volumen mayor y suficiente para llenar los estómagos de las lagartijas con pocos individuos. Otro aspecto de las hormigas es que son insectos sociales terrestres (Jaffé 1993) que pueden encontrarse con mayor abundancia en espacios reducidos (viven en nidos de manera agrupada) que el resto de ítems, lo que las hace más fáciles de capturar, reduciendo de esta manera sus costes energéticos en la búsqueda de otras presas (Gutiérrez 1998, Álvarez et al. 2014). La obtención de alimento implica que el gasto de energía en los organismos sea mayor, por lo que tratan de buscar alimento que rinda el máximo de energía por unidad de consumo (Pianka 1982).

La baja amplitud de nicho trófico de $M$. occipitalis en esta área de estudio $\left(B_{\text {est }}=0.13\right)$ está directamente relacionado con el alto consumo de himenópteros. Si las hormigas son la mayor oferta de alimento para esta especie, entonces, estarían contribuyendo efectivamente con la baja amplitud de nicho porque la amplitud de nicho trófico tiende a disminuir conforme aumenta la oferta de alimento (Dunham 1983).

Los coleópteros son un grupo importante y común en la dieta de algunas especies del género Microlophus de la costa peruana (Pérez \& Balta 2007). En M. occipitalis, los coleópteros fueron el segundo ítem alimenticio que fue consumido con alta frecuencia. Su presencia en la dieta de $M$. occipitalis se debería a que estos insectos predominan en plantas herbáceas por ser esencialmente fitófagos (Barrientos 1988, Coronado \& Márquez 1999, Fernández et al. 2014).

Las adaptaciones morfológicas necesarias para el aprovechamiento de vegetales en la dieta de la familia Tropiduridae ha sido documentado (Toyama 2017), lo que explicaría la presencia de diferentes estructuras vegetales en la dieta de muchas de sus especies (Cooper \& Vitt 2002, Carvalho et al. 2007) incluyendo a las del género Microlophus (Campos 1999, Vargas 2000, Pérez \& Balta 2007, Pérez et al. 2015, Toyama 2017). Pough (1973), argumentó que el consumo de materia vegetal por parte de saurios, se debe a que la obtención de los insectos tiene un alto costo energético y la depredación puede reducir las áreas de actividad, por lo que pueden acceder con mayor facilidad a la disponibilidad de los vegetales presentes en dichos espacios. Sin embargo, el consumo de vegetales en $M$. occipitalis puede deberse a su historia filogenética, es decir, que la herbivoría se presentó tempranamente en la evolución del grupo como plantearon Lemus y Bellinger (1996) para Sceloporus torquatus (Squamata, Phrynosomatidae).

Microlophus occipitalis presentó una baja amplitud de nicho lo que indicaría que esta especie tiende a ser especialista. Esta especialización estaría explicada por tener una dieta mayoritariamente carnívora, especialmente hormigas (Hymenopteros), lo que contrasta notablemente con lo reportado para otras especies del mismo género (Dixon \& Wright 1975, Vargas 2000, Catenazzi 2002, Pérez \& Balta 2007, Pérez et al. 2008, Quispitúpac \& Pérez, 2009), quienes plantean que el género básicamente es generalista. Sin embargo, la dieta de $M$. occipitalis también incluyó materia vegetal aunque en un bajo porcentaje, lo que permite plantear que esta materia vegetal llegó al estómago por ingestión accidental, dado que Espinoza et al. (2004) consideran que para que una especie sea herbívora el consumo debe ser del orden del $70-100 \%$.
El índice de Levins es un índice de diversidad que al igual que otros índices de diversidad, expresa valores bajos cuando la dominancia es alta. Los índices basados en la dominancia son parámetros inversos al concepto de uniformidad o equidad de la comunidad, es decir, toman en cuenta la representatividad de las especies con mayor valor de importancia sin evaluar la contribución del resto de las especies (Moreno 2001). Por lo tanto, considerando los tratados de biodiversidad de Magurran (1988) y Moreno (2001), a mayor dominancia, menor equidad en la distribución de individuos por especie, o lo que sería lo mismo afirmar que, a mayor dominancia en uno de los ítems alimenticios más baja será la amplitud de nicho.

La disponibilidad y abundancia de recursos en un lugar determinado responde a factores climáticos, por lo que lugares con cambio estacional marcado, presentarán variación en la disponibilidad de dichos recursos, entre ellos los de hábitat y los de tipo alimenticio (Altamirano-Álvarez 2014). Este estudio se realizó durante la temporada de primavera-verano por lo que el alcance de los resultados podrían variar con respecto al otońoinvierno y localidad de estudio.

\section{Agradecimientos}

A Leticia Cruzado, Jorge Cobeñas, Gino Juárez, Juan López, Alex Bermejo, Wilder Cava, Milagros Pérez, Jorge Carrera, Francisca Torres y José Domínguez por el apoyo en el trabajo de campo. A los revisores anónimos que con sus observaciones contribuyeron a mejorar el manuscrito.

\section{Literatura citada}

Altamirano T.A., S.M. Soriano \& A.A. Gelover. 2001. Hábitos alimenticios de Bufo valliceps bajo distintas condiciones; con aportación al conocimiento de la ecología alimenticia de Bufo marinus y Bufo marmoreus. Revista De Zoología, FES-Iztacala, UNAM 12: 28-32.

Altamirano-Álvarez T., E. Pérez-Sánchez, M. Soriano, A. Bernal-García \& N. Miranda-González. 2014. Determinación del espectro alimentario de la lagartija Sceloporus torquatus (Reptilia: Phrynosomatidae) a través de excretas. Revista de Zoología 25: $10-17$.

Angulo N. 1955. Formaciones vegetales de la costa norte del Perú. Universidad Nacional de Trujillo - Perú.

Bakken G. 1989. Arboreal perch properties and the operative temperature experienced by small animals. Ecology 70: 922-930. https://doi.org/10.2307/1941359

Bartholomew G. 1982. Physiological control of body temperature. En: Gans C \& Pough FH (eds), Biology of Reptilia, 12C: 167-211. Academic Press, London, UK.

Barrientos J. 1998. Bases para un curso práctico de entomología. Asociación española de Entomología. CIBIO Centro Iberoamericano de la Biodiversidad. Universidad Autónoma de Barcelona. Servei de Publicacions. Manuales de la Universidad Autónoma de Barcelona 41.

Brennan R. 2010. Un estudio ecológico de las lagartijas del Valle Seco de Buenavista y de los Valles Húmedos de la Josefina y Salango. Independent Study Project (ISP). Collection 828.

Campos V. 1999. Estimación de los parámetros poblacionales de los Tropiduridae y Teiidae del cerro Cabezón - Trujillo, de enero a mayo de 1999. Tesis para optar el título profesional de Biólogo. Universidad Nacional de Trujillo.

Carvalho A., H. Da Silva. A. Araujo, R. Alvés \& R. Da Silva. 2007. Feeding ecology of Tropidurus torquatus (Wied) (Squamata, Tropiduridae) in two areas with different degrees of conservation in Marambaia Island, Rio de Janeiro, Southeastern Brazil. Revista Brazileira de Biología 24(1): 222-227. http:// dx.doi.org/10.1590/S0101-81752007000100029 
Catenazzi A. 2002. Evaluación de la técnica de lavado del estómago para determinar la dieta de las lagartijas Microlophus peruvianus y $M$. theresiae. Informe técnico final para INRENA correspondiente a la Autorización $\mathrm{N}^{\circ}$ 016-2001-INRENAJ-DGANPFS-DCFS.

Carothers J., P. Marquet \& F. Jaksic. 1998. Thermal ecology of a Liolaemus lizard assemblage along an Andean altitudinal gradient in Chile. Revista Chilena de Historia Natural 71: 39-50.

Carrillo N. \& J. Icochea. 1995. Lista taxonómica preliminar de los reptiles vivientes del Perú. Publicaciones del Museo de Historia Natural UNMSM (A) 49: 1-27.

Clauser C. \& E. Huenges. 1995. Thermal conductivity of rocks and minerals. En: Rocks Physics and Phase Relations: a handbook of physical constants, AGU Reference Shelf. https:// doi.org/10.1029/RF003p0105

Cooper W. \& J. Vitt. 2002. Distribution, extent and evolution of plants consumption by Lizards. J. Zool. 257 (4): 487-517. https://doi.org/10.1017/S0952836902001085

Coronado P. \& D. Márquez. 1999. Introducción a la Entomología. Morfología y Taxonomía de los Insectos. Ed. México: UTEHA: Noriega.

Dixon J. \& J. Wright. 1975. A review of the lizards of the iguanid genus Tropidurus in Peru. Contribution in Science The Natural History Museum of Los Angeles 271: 1-39.

Dunham A. 1983. Realized niche overlap, resource abundance, and intensity of interspecific competition. pp. 261-280. En: Huey, R. E. Pianka E. R. y Schoener T. W. (eds.), Lizard Ecology. Studies of a Model Organism. Harvard University Press, Cambridge. 261-280 pp.

Eakin R. 1957. Use of Cooper wire in noosing lizard. En: Manzanilla J. \& Péfaur J. 2000. Consideraciones Sobre Métodos Y Técnicas de Campo Para el estudio de Anfibios y Reptiles. Rev. Ecol. Lat. Am. 7(1-2), 17-30.

Espinoza R E., J.J. Wiens, C.R. Tracy \& D.B. Wake. 2004. Recurrent evolution of herbivory in small, cold-climate lizards: Breaking the ecophysiological rules of reptilian herbivory. PNAS 101 (48): 16819-16824. https://doi.org/10.1073/ pnas.0401226101

ESRI (Environmental Systems Research Institute Inc.). 2002. Arc View Gis, version 3.3. Nueva Delhi, India.

Fernández I., M. Favila \& G. López. 2014. Composición, riqueza y abundancia de coleópteros (Coleoptera) asociados a bosques semideciduos y vegetaciones ruderales en la Sierra del Rosario, Cuba. Boletín de la Sociedad Entomológica Aragonesa 54: 329-339

Ferreyra R. \& O. Tovar. 1955. Las gramíneas (Poaceae) del Perú. Mus. Hist. Nat. Javier Prado. Lima, Ser. B. Bot. I. No 10, 14.

Gutiérrez G. 1998. Estrategias de forrajeo. En R. Ardila, W. López, A.M. Pérez, R. Quińones \& F. Reyes (Eds.). Manual de Análisis Experimental del Comportamiento. Págs. 359-381. Madrid: Librería Nueva.

Heath J. E. 1964. Reptilian thermoregulation: evaluation of field studies. Science 146: 784- 785. https://doi.org/10.1126/ science.146.3645.784

Heise P.J. 1998. Phylogeny and biogeography of Galapagos lava lizards (Microlophus) inferred from nucleotide sequence variation in mitochondrial DNA. Ph.D. Dissertation, The University of Tennessee, Knoxville, Tennessee.

Huey R. 1982. Temperature, physiology and the ecology of reptiles, pp. 25-91, in: Gans, C. \& Pough, F. H. (eds.), Biology of the Reptilia. Vol. 12. P. Academic Press, New York.

Huey R. \& E. Pinka. 1977. Natural selection for juvenile lizards mimicking noxious beetles. Science 195: 201-203. https:// doi.org/10.1126/science. 831272

Jaffé K. 1993. El mundo de las hormigas. Baruta, Venezuela. Ediciones Equinoccio. Universidad Simón Bolívar.

Johnson D. 1999. The insignificance of statistical significance testing. Journal of Wildlife Management, 63:763-772. https://doi. org $/ 10.2307 / 3802789$

Jordán J.C. \& J. Pérez. 2012. Thermal ecology of Microlophus occipitalis (Sauria: Tropiduridae) in the plain dry forest of Tumbes, Peru. Rev. peru. biol. 19:97-99. https://doi.org/10.15381/ rpb.v19i1.794
Krebs C. 1989. Ecological methodology. Harper \& Row, Nueva York.

Labra A., M. Vidal, R. Solís \& M. Penna. 2008. Ecofisiología de Anfibios y Reptiles. En: Vidal, M \& Labra, A., editores. Herpetología de Chile. Chile: GráficAndes 2008. 483-516p.

Legler J. \& L. Sullivan. 1979. The application of stomach-flushing to lizards and anurans. Herpetologica 35:107-110.

Magurran A.E. 1988. Ecological diversity and its measurement. Princeton University Press, New Jersey. https://doi. org/10.1007/978-94-015-7358-0

Manly B., L. McDonald, D. Thomas, T. McDonald \& W. Erickson. 2003. Resource selection by animals - Statistical design and Analysis for field studies. Second edition London: Kluwer academic publishers.

Martin J. 1992. Consecuencias de la pérdida de la cola en la lagartija serrana (Lacerta monticola). Tesis para optar el grado de Doctor. Universidad Complutense de Madrid (España).

Martin A. \& Hockey P. 1993. The effectivess of stomach-flushing in assessing wader diets. Wader Study Group Bull.67: 79-80.

Moreno, C. 2001. Métodos para medir la biodiversidad. M\&T-Manuales y Tesis SEA, vol. 1. Zaragoza, España.

Mostacero L., C. Mejía \& P. Peláez. 1996. Fitogeografía del Norte del Perú. Ed. CONCYTEC Lima-Perú.

Pearson O. 1977. The effect of substrate and of skin color on thermoregulation of a lizard. Comparative Biochemistry \& Physiology A 58: 353-358. https://doi.org/10.1016/03009629(77)90154-2

Pérez J. \& K. Balta. 2007. Ecología de la comunidad de saurios diurnos de la Reserva de Paracas Ica Perú. Rev. Per Biol 13(3): 169179. http://dx.doi.org/10.15381/rpb.v13i3.2334

Pérez J., K. Balta, R. Ramírez \& D. Susanibar. 2008. Succinea peruviana (Gastropoda) en la dieta de la lagartija de las lomas Microlophus tigris (Sauria) en la Reserva Nacional de Lachay, Lima, Perú. Rev. Per. Biol. 15(1): 109-110. http://dx.doi. org/10.15381/rpb.v15i1.1685

Pérez J., E. Fuentes \& J. Jordán. 2015. Dieta de la lagartija de los gramadales Microlophus thoracicus icae en el valle del río Ica, Perú. Rev. Per. Biol 22(2): 233 - 238. https://doi. org/10.15381/rpb.v22i2.11357

Pianka E. R. 1982. Ecología evolutiva. Omega.España.

Pough F.H. 1973. Lizard energetics and diet.The Ecological Society of America: Ecology. 54 (4): 837-844.

Quispitúpac E. \& J. Pérez. 2009. Dieta de la lagartija de la playa Microlophus peruvianus (Reptilia: Tropiduridae) en la playa Santo Domingo, Ica, Perú. Rev. peru. biol. 15(2): 129-130. http://dx.doi.org/10.15381/rpb.v15i2.1739

R Core Team. 2009. R: A language and environment for statistical computing. R Foundation for Statistical Computing, Vienna, Austria. < URL https://www.R-project.org/>. Acceso $15 / 10 / 2010$.

Rivas J., C. Molina \& T. Ávila. 1996. Non-flushing stomach wash technique for large lizards. Herp. Rev. 27(2):72-73.

Sarria, M., \& Silva, L. 2004. Las pruebas de significación estadística en tres revistas biomédicas: una revisión crítica. Revista Panamericana de Salud Pública, 15:300-306. https://doi. org/10.1590/S1020-49892004000500003

Toyama K. 2017. Ecomorfología de dieta de la lagartija de los gramadales Microlophus thoracicus (Tropiduridae). Tesis para optar el grado de Bachiller en Ciencias con mención en Biología. Facultad de Ciencias y Filosofía. Universidad Peruana Cayetano Heredia (Perú).

Vargas C. 2000. Amplitud de nicho de Plesiomicrolophus koeckeorum Mertens (1956) en las Lomas de Poroto, Trujillo. Febrero - julio del 2000. Tesis para optar el título profesional de Biólogo. Facultad de Ciencias Biológicas, Universidad Nacional de Trujillo (Perú).

Venegas P. 2005. Herpetofauna del Bosque Seco Ecuatorial de Perú: taxonomía, ecología y biogeografía. Zonas Áridas 9: 9-26.

Viera R. 2007. Densidad poblacional de reptiles en el Bosque Seco de Chapaira, Castilla-Piura entre los meses de enero- junio 2007. Tesis para optar el título profesional de Biólogo. Facultad de Ciencias, Universidad Nacional de Piura (Perú).

Wareham D.C. 2005. Elsevier's dictionary of herpetological and related terminology. Elsevier. 
Watkins G.G. 1997. Inter-sexual signalling and the function of female coloration in the tropidurid lizard Microlophus occipitalis. Animal Behaviour 53(4):843-852. https://doi.org/10.1006/ anbe. 1996.0350

Watkins G.G. 1998. Function of a secondary sexual ornament: The crest in the South American iguanian lizard Microlophus occipitalis (Peters, Tropiduridae). Herpetológica 54(2), 161-169.
Weberbauer A. 1945. El mundo vegetal de los andes peruanos. Nueva edición. Edit. Estación Experimental Agrícola de La Molina. Dirección de Agricultura. Ministerio de Agricultura. Lima - Perú.

Wiens J.A. 1984. Resource systems, populations, and communities. Pages 397-436 in P. W. Price, C. N. Slobodchikoff, and W. S. Gaud, eds. A new ecology: novel approaches to interactive systems. John Wiley and Sons, New York, N.Y. 